\title{
Temporal Diffeomorphic Free-Form Deformation for Strain Quantification in 3D-US Images
}

\author{
Mathieu De Craene ${ }^{1,2}$, Gemma Piella ${ }^{1,2}$, Nicolas Duchateau ${ }^{1,2}$, Etel Silva ${ }^{4}$, \\ Adelina Doltra ${ }^{4}$, Hang Gao ${ }^{5}$, Jan D'hooge ${ }^{5}$, Oscar Camara ${ }^{1,2}$, Josep Brugada ${ }^{4}$, \\ Marta Sitges ${ }^{4}$, and Alejandro F. Frangi ${ }^{1,2,3}$ \\ Center for Computational Imaging \& Simulation Technologies in Biomedicine \\ (CISTIB) \\ ${ }^{1}$ Information and Communication Technologies Department, \\ Universitat Pompeu Fabra, Barcelona, Spain \\ 2 Networking Center on Biomedical Research - CIBER-BBN, Barcelona, Spain \\ ${ }^{3}$ Institucio Catalana de Recerca i Estudis Avancats, Barcelona, Spain \\ ${ }^{4}$ Hospital Clínic; IDIBAPS; Universitat de Barcelona, Spain \\ ${ }^{5}$ Department of Cardiovascular Diseases, Cardiovascular Imaging and Dynamics, \\ Katholieke Universiteit Leuven, Belgium
}

\begin{abstract}
This paper presents a new diffeomorphic temporal registration algorithm and its application to motion and strain quantification from a temporal sequence of 3D images. The displacement field is computed by forward eulerian integration of a non-stationary velocity field. The originality of our approach resides in enforcing time consistency by representing the velocity field as a sum of continuous spatiotemporal BSpline kernels. The accuracy of the developed diffeomorphic technique was first compared to a simple pairwise strategy on synthetic US images with known ground truth motion and with several noise levels, being the proposed algorithm more robust to noise than the pairwise case. Our algorithm was then applied to a database of cardiac 3D+t Ultrasound (US) images of the left ventricle acquired from eight healthy volunteers and three Cardiac Resynchronization Therapy (CRT) patients. On healthy cases, the measured regional strain curves provided uniform strain patterns over all myocardial segments in accordance with clinical literature. On CRT patients, the obtained normalization of the strain pattern after CRT agreed with clinical outcome for the three cases.
\end{abstract}

\section{Introduction}

Quantification of cardiac motion and strain provides insight about cardiac function by estimating how a given pathology affects global or local contractility of the myocardium. In clinical routine, motion and strain are usually derived from ultrasound (US) images for which 3D acquisitions are now currently available with sufficient spatiotemporal resolution for characterizing motion and strain. Nonetheless, 3D-US images have lower signal-to-noise ratio (SNR) and temporal resolution than the $2 \mathrm{D}$ ones, thus making their processing more challenging.

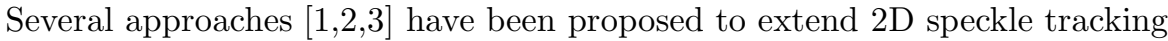
techniques to $3 \mathrm{D}$ and to recover myocardial motion from a sequence of $3 \mathrm{D}-\mathrm{US}$

T. Jiang et al. (Eds.): MICCAI 2010, Part II, LNCS 6362, pp. 1-8, 2010.

(C) Springer-Verlag Berlin Heidelberg 2010 
images. One of the main drawbacks of these techniques based on pairwise registrations is that they do not make use of the temporal information embedded in the 3D-US sequences. Parametric spatiotemporal models providing continuous and smooth transformations were proposed by Ledesma-Carbayo et al. [4] to exploit temporal information in 2D-US image sequences. However, ensuring temporal smoothness of the displacement field does not render properly temporal consistency, i.e. that the displacement field at each time point is related to all the previous times.

Diffeomorphic registration algorithms ([56] among others) ensure a continuous, differentiable and with continuous inverse, correspondence between the features to register. Thus, they are particularly well suited to handle medical image sequences as they conserve the topology and the orientation of the observed anatomical structures along time. By integrating a velocity field over time, they provide an elegant way of encoding temporal consistency.

This concept was applied by Khan et al. 7] to monitor growth processes by extending the LDDMM [5] image registration algorithm. Velocities were computed using a dense grid, which did not guarantee their spatiotemporal continuity, unlike parametric registration methods. Moreover, a regularization term added to the image similarity metric involved a smoothing kernel that enforced the spatial continuity of the computed velocities. The temporal continuity of the velocities was then fully conditioned by the conservation of the topology of the observed features along the image sequence. This assumption does not hold for noisy image sequences, such as cardiac US images. Similar concepts were applied to 2D contours and 3D shapes by Durrleman et al. [8]. However, while the computational cost of dense velocity fields is acceptable for sparse topologies, its extension to dense volumetric spatiotemporal data remains critical.

In this paper, we propose a diffeomorphic registration algorithm that models velocities continuously in time and space. We refer to our approach as Temporal Diffeomorphic Free-Form Deformation (TDFFD) algorithm. We extend the popular parametric FFD registration technique [6] by summing spatiotemporal $\mathrm{B}$-Spline kernels to model the $3 \mathrm{D}+\mathrm{t}$ velocity field. One of the main advantages of our approach is the enforcement of the continuity of the velocity field by using a continuous parametric representation. As a result, the velocity and displacement fields can be computed at any time within the temporal interval captured by the image sequence. The advantage of applying such transformation model for strain quantification purposes is demonstrated here on synthetic and real 3D-US image sequences, with the underlying objective of accurately estimating the impact of Cardiac Resynchronization Therapy (CRT) on 3D strain.

\section{Registration Algorithm and Strain Computation}

In this paper, we consider a sequence of $N$ images $\left\{I_{n}, n=1 \ldots N\right\}$, each image being defined on a spatial domain $\Omega \subset \mathbb{R}^{d}$ where $d=3$ stands for the spatial dimension. Each image $I_{n}$ is associated to a time instant $t_{n} \in[0, T]$, with $T>$ $0 \in \mathbb{R}$. The purpose of the registration algorithm is to solve for the diffeomorphic 
mapping $\varphi: \Omega \times[0, T] \rightarrow \mathbb{R}^{d}$ that relates any point $\mathbf{x}$ in the Eulerian space of coordinates of the first image in the sequence (here taken by convention as $t=0)$ to a continuous time $t \in[0, T]$.

The temporal dimension is introduced into the diffeomorphic registration problem by relating the mapping $\varphi$ at any time $t \in[0, T]$ to a time-varying velocity field. In this paper, the velocity field is represented as a sum of spatiotemporal kernels. The B-Spline velocity weights given to all kernels are concatenated in a vector of parameters $\mathbf{p}$, the velocity being then denoted as $\mathbf{v}(\mathbf{x}, t, \mathbf{p})$ and computed as

$$
\mathbf{v}(\mathbf{x}, \mathbf{p}, t)=\sum_{\tau} \sum_{\mathbf{c}} \beta\left(\frac{t-t^{\tau}}{\Delta^{\tau}}\right) B\left(\frac{\mathbf{x}-\mathbf{x}^{c}}{\boldsymbol{\Delta}^{\mathbf{c}}}\right) \mathbf{p}^{\tau, \mathbf{c}}
$$

where $B(\cdot)$ is the $3 \mathrm{D}$ tensor product of $1 \mathrm{D}$ cubic B-spline kernels $\beta(\cdot)$, defined on a sparse grid of $4 \mathrm{D}$ control points, being $\mathbf{c}$ the spatial index, $\tau$ the temporal index, and $\left(\boldsymbol{\Delta}^{\mathbf{c}}, \Delta^{\tau}\right)$ the width of the kernels in each dimension. Hence, the $\boldsymbol{\varphi}$ mapping is obtained as

$$
\boldsymbol{\varphi}(\mathbf{x}, t, \mathbf{p})=\mathbf{x}+\int_{0}^{t} \mathbf{v}(\boldsymbol{\varphi}(\mathbf{x}, \tau, \mathbf{p}), \tau, \mathbf{p}) \mathrm{d} \tau .
$$

Forward eulerian integration scheme. The transport equation for computing $\varphi$ in Eq. 2 is solved numerically using a forward Euler integration scheme in which the continuous integral is replaced by a discrete summation. The continuous time interval is now split into a collection of $t_{k} \in[0, T]$ values where the time increment $\Delta t_{k}$ between consecutive time-steps is adapted to ensure invertibility as described in the next subsection. Using this discretization, Eq. 2 can be approximated by

$$
\boldsymbol{\varphi}\left(\mathbf{x}, t_{n}, \mathbf{p}\right)=\mathbf{x}+\sum_{k=0}^{n-1} \mathbf{v}\left(\boldsymbol{\varphi}\left(\mathbf{x}, t_{k}, \mathbf{p}\right), t_{k}, \mathbf{p}\right) \Delta t_{k},
$$

If we define $\mathbf{x}_{k}(\mathbf{p}) \doteq \boldsymbol{\varphi}\left(\mathbf{x}, t_{k}, \mathbf{p}\right), t_{k} \doteq \sum_{l=0}^{k-1} \Delta t_{l}$, and $\mathbf{v}_{k-1}(\mathbf{p}) \doteq \mathbf{v}\left(\mathbf{x}_{k-1}, t_{k-1}, \mathbf{p}\right)$ then we can recursively write $\mathbf{x}_{k}$ as follows:

$$
\mathbf{x}_{k}(\mathbf{p})=\mathbf{x}_{k-1}(\mathbf{p})+\mathbf{v}_{k-1}(\mathbf{p}) \Delta t_{k-1} .
$$

Adaptive time-step computation. The integration of Eq.2 using the discrete approximation of Eq. 4 requires to select a time-step sufficiently small for ensuring accurate computation and invertibility of the mapping $\boldsymbol{\varphi}$. In our method, we start with a uniform sampling of the time interval $[0, T]$, arbitrarily chosen as half of the temporal spacing of the image sequence. To ensure invertibility, one needs to consider the Jacobian of the mapping $\mathbf{x}_{n}(\mathbf{p})$, here denoted as $\mathbf{D} \mathbf{x}_{n}(\mathbf{p})$ and computed from Eq. 4 using

$$
\mathbf{D} \mathbf{x}_{n}(\mathbf{p})=\prod_{k=0}^{n-1}\left(\mathbf{I}+\mathbf{D} \mathbf{v}_{k}(\mathbf{p}) \Delta t_{k}\right) \doteq \prod_{k=0}^{n-1} \Delta \boldsymbol{\varphi}_{k}
$$

where I stands for the identity matrix. This Jacobian must be positive definite everywhere to ensure invertibility of the transformation. A necessary condition 
for this is to have $\operatorname{det}\left(\mathbf{D} \mathbf{x}_{n}(\mathbf{p})\right)>0$ for all $\mathbf{x} \in \Omega$. Computing the product over $k$ of all $\operatorname{det}\left(\Delta \boldsymbol{\varphi}_{k}\right)$ gives the determinant of the Jacobian matrix in Eq. 5. When a negative value of $\operatorname{det}\left(\Delta \varphi_{k}\right)$ is detected, the value of $\Delta t_{k}$ is reduced by a factor 2 until the obtention of a positive determinant.

Similarity metric and non-linear optimization. 3D-US images are characterized by a speckle spatial distribution inside the myocardial wall that is conserved along time. The Mean Squared Error (MSE) appears therefore as a good fit for capturing the optimal set of B-Spline velocity weights $\mathbf{p}$ from Eq. 1 . Similarity is measured between the first image in the sequence and all the consecutive frames according to

$$
M S E(\mathbf{p})=\sum_{n=0}^{N-1} \int_{\Omega}\left(I_{0}(\mathbf{x})-I_{n}\left(\mathbf{x}_{n}(\mathbf{p}), t_{n}\right)\right)^{2} \mathrm{~d} \mathbf{x}
$$

Since the number of parameters characterizing the transformation is large, and the metric is explicitly differentiable, gradient-based optimization methods are well indicated for minimizing Eq. 6. In this paper, the L-BFGS-B method was used, which searches the optimum according to the gradient and a low-rank approximation of the Hessian of the metric. For computing the total derivative of Eq. 6] with respect to the weights $\mathbf{p}$ of velocity kernels, we need the following derivative:

$$
\frac{\mathrm{d} \mathbf{x}_{n}}{\mathrm{~d} \mathbf{p}}=\frac{\mathrm{d} \mathbf{v}_{n-1}}{\mathrm{~d} \mathbf{p}} \Delta t_{n-1}+\frac{\mathrm{d} \mathbf{x}_{n-1}}{\mathrm{~d} \mathbf{p}} \text { where } \frac{\mathrm{d} \mathbf{v}_{n-1}}{\mathrm{~d} \mathbf{p}}=\frac{\partial \mathbf{v}_{n-1}}{\partial \mathbf{x}_{n-1}} \frac{\mathrm{d} \mathbf{x}_{n-1}}{\mathrm{~d} \mathbf{p}}+\frac{\partial \mathbf{v}_{n-1}}{\partial \mathbf{p}} .
$$

Hence, $\mathrm{d} \mathbf{x}_{n} / \mathrm{d} \mathbf{p}$ can be obtained from the following recursive equation:

$$
\frac{\mathrm{d} \mathbf{x}_{n}}{\mathrm{~d} \mathbf{p}}=\frac{\mathrm{d} \mathbf{x}_{n-1}}{\mathrm{~d} \mathbf{p}}\left(\mathbf{I}+\mathbf{D} \mathbf{v}_{n-1} \Delta t_{n-1}\right)+\frac{\partial \mathbf{v}_{n-1}}{\partial \mathbf{p}} \Delta t_{n-1},
$$

where $\mathbf{D}$ is the Jacobian on all spatial dimensions (i.e., $(\mathbf{D v})_{i j}=\partial \mathbf{v}_{i} / \partial \mathbf{x}_{j}$ ).

Strain computation. The Cauchy strain tensor is estimated directly using the spatial derivatives of the displacement field from Eq. 5. obtained at any spatiotemporal location using Eq. 4. The Cauchy strain tensor is then computed as

$$
\boldsymbol{\sigma}\left(\mathbf{x}, t_{n}\right)=\frac{1}{2}\left(\left(\mathbf{D} \mathbf{x}_{n}\right)^{t} \mathbf{D} \mathbf{x}_{n}-\mathbf{I}\right) \text {. }
$$

Strain is obtained along a specific direction $\mathbf{h}$ using $\sigma_{\mathbf{h}}(\mathbf{x}, t)=\mathbf{h}^{t} \cdot \boldsymbol{\sigma}(\mathbf{x}, t) \cdot \mathbf{h}$. The $\mathbf{h}$ directions considered here are the three vectors (circumferential, longitudinal, radial) of a parabolic coordinate system related to the anatomy of the left ventricle.

\section{$3 \quad$ Experiments}

The proposed registration algorithm was applied to three different synthetic datasets to evaluate its accuracy and was then applied to clinical routine 3D-US 

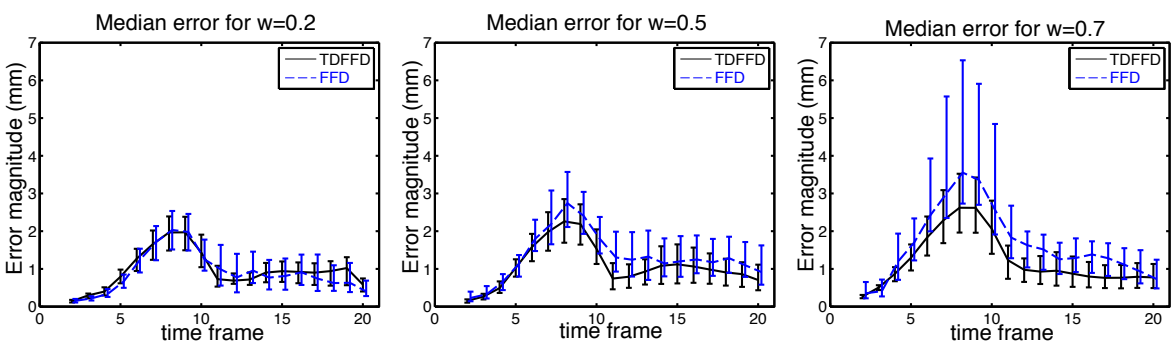

Fig. 1. Median value of error magnitude on the displacement field for the FFD (plotted in blue dashed) and the TDFFD algorithm (black). Vertical bars indicate the second and third quartiles. Three levels of noise are considered: $w=0.2$ (left), $w=0.5$ (center) and $w=0.7$ (right).

images. First, a simple pairwise strategy and the proposed temporally consistent scheme were compared taking a ground truth deformation from simulated US data as reference. Then, strain curves were estimated from 3D-US sequences for 8 volunteers and 3 CRT patients before the therapy and at 12-months follow-up.

\subsection{Registration Accuracy on Simulated US Data}

Elen et al. 2] simulated Left Ventricle (LV) deformation in which the LV was represented as a thick-walled ellipsoid with physiologically relevant end-diastolic dimensions. A simplified kinematic model with an ejection fraction of $60 \%$ over a cardiac cycle was used to generate the ground truth displacement field. We used this ground truth data to evaluate the accuracy of the proposed algorithm and compare it to a pairwise registration strategy at different noise levels. Various signal to noise ratios were generated by adjusting intensities inside and outside the myocardial wall using a weight $w(w=0.2, w=0.5$ and $w=0.7$ in this paper $)$. Fig. 11 shows the median magnitude and dispersion of the difference between the ground truth displacement field and the ones given by two algorithms: a pairwise FFD (see reference in [6]) and the temporal diffeomorphic FFD registration (TDFFD) algorithms. This error was computed over the entire myocardial wall. FFD pairwise registration was performed between each image and the first image in the sequence, taking the chain of previously computed transformations as bulk transformation. For the two algorithms, the B-Spline grid had an initial resolution of three control points in the longitudinal direction and five in the the two transverse directions. This resolution was then refined twice by a factor 2. For the lowest amount of noise, there was no substantial difference between FFD and TDFFD strategies. However, as the signal to noise ratio decreased, the TDFFD algorithm proved to be more robust and produced smaller errors (maximal median error of $2.6 \mathrm{~mm}$ for TDFFD and $3.6 \mathrm{~mm}$ for pairwise FFD). The dispersion was also clearly reduced when using the TDFFD algorithm for all noise levels, as observed in Fig. 1]. For $w=0.7$, the upper limit of the third quartile goes from $6.6 \mathrm{~mm}$ using pairwise FFD to $3.5 \mathrm{~mm}$ using TDFFD. 

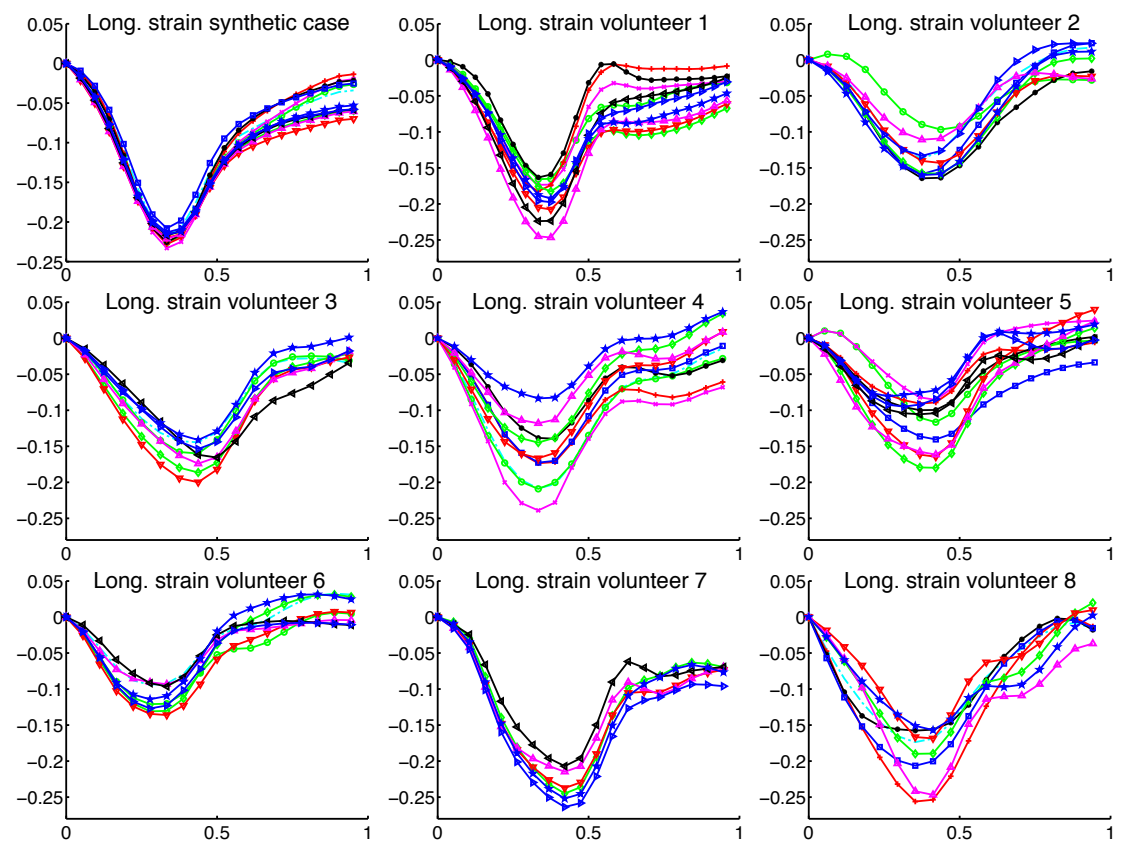

Fig. 2. Longitudinal strain quantified for the synthetic case and 8 healthy volunteers in basal and mid segments. The AHA segments are labelled according to the following: - Basal anterior (1), ○ Basal anteroseptal (2), $\times$ Basal inferoseptal (3), + Basal inferior (4), * Basal inferolateral (5), $\square$ Basal anterolateral (6), $\diamond$ Mid anterior (7), $\nabla$ Mid anteroseptal (8), $\triangle$ Mid inferoseptal (9), $\triangleleft$ Mid inferior (10), $\triangleright$ Mid inferolateral (11), $\star$ Mid anterolateral (12). Color version of this figure available at http://bit.ly/miccai10.

\subsection{Experiments on Clinical Datasets}

Data acquisition. We acquired 3D echocardiographic sequences in an apical view for two populations, using a General Electric (Milwaukee, WI, USA) Vivid 7 device. The first population was made up of 8 healthy volunteers (aged $31 \pm$ 6 years), and the second population was composed of 3 CRT patients (aged 61 \pm 8 years), who were all clinical responders to CRT. The average number of images per cardiac cycle was of 17.8 for the healthy subjects and 18.3 for the CRT patients. The pixel spacing was on average of $0.9 \times 0.6 \times 0.9 \mathrm{~mm}^{3}$ for the healthy volunteers and $1.0 \times 0.7 \times 1.0 \mathrm{~mm}^{3}$ for the CRT patients.

Strain in healthy volunteers. Fig. 2 shows the recovered longitudinal strain curves for the database of 8 healthy subjects at mid and basal segments of the American Heart Association (AHA). The segments either not totally included in the field of view of the 3D-US images or suffering from typical image artifacts (non visibility of lateral wall, reflections of surrounding anatomical structures, lower spatial resolution on the lateral sides of the sector) of 3D-US acquired 

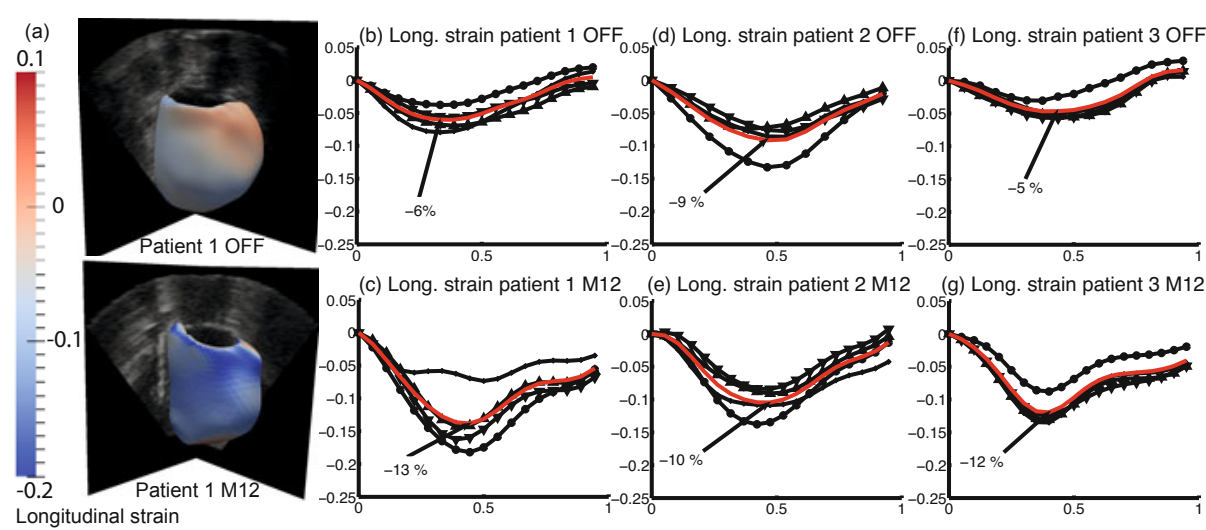

(c) Long. strain patient $1 \mathrm{M} 1$

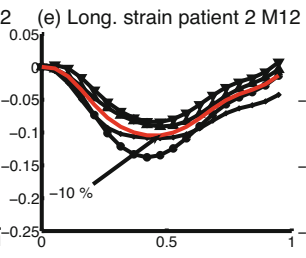

(g) Long. strain patient $3 \mathrm{M} 12$
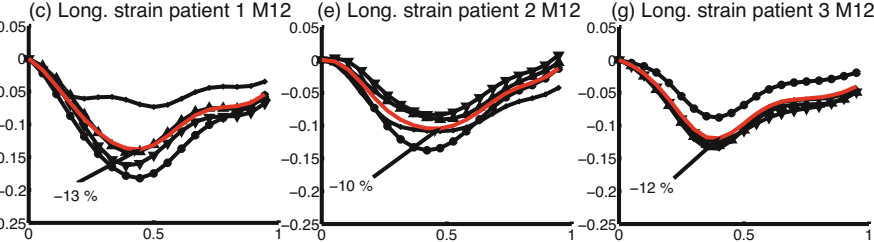

Fig. 3. (a) Longitudinal strain as a colormap at end of systole before CRT (top) and at twelve months follow-up (bottom) for Patient 1. (b-g) Longitudinal strain curves in septal regions before (OFF) and at 12 months follow-up (M12) for Patients 1 to 3. Color version of this figure available at http://bit.ly/miccai10.

clinically, were excluded from the analysis. The recovered strain curves showed a similar pattern in all volunteers, in good agreement with clinical literature [9]. The average peak systolic strain was of $-16.3 \pm 4.7 \%$. This value is close the $-17.5 \pm 4 \%$ reported in [9] and obtained from tagged MRI images. Different phases of diastole such as the isovolumetric relaxation and the atrial contraction (acceleration of the strain at the end of the diastole) periods can be distinguished in cases with higher temporal image resolution such as Volunteers 1 and 4.

Strain before and after CRT. The three CRT patients processed in this paper had dilated geometry before implantation, thus the LV did not fit entirely in the field of view. Therefore, strain was only quantified in the septal regions that usually have the best image quality. Fig. 3 shows longitudinal strain plotted using a color map for the first patient and its temporal evolution per septal segment for all the patients. Average strain curves are shown in red and the peak systolic strain value before and after CRT is indicated by an arrow. Patients 1 and 3 showed a significant improvement in peak systolic strain after CRT that correlated well with an important reverse remodeling observed in these two patients at the 12-month follow-up (reduction of end-systolic volume of $27.7 \%$ and $51.0 \%$, respectively). For Patient 2, no substantial change in peak strain value was observed from the strain curves. This patient had lower reverse modeling as observed at the follow-up with a reduction of end-systolic volume of $16.9 \%$. This value is very close to $15 \%$, which is the threshold used at our institution for defining positive CRT response.

\section{Conclusion}

This paper presents a new diffeomorphic registration method ensuring temporal consistency of the resulting deformation fields, which can be particularly 
useful in image sequences with substantial amount of noise and artifacts. This algorithm was applied for the quantification of strain in 3D US using synthetic datasets, and a set of healthy subjects and CRT patients. Experiments on synthetic US datasets proved an improved robustness and accuracy at high noise levels compared to classical pairwise approaches. On healthy volunteers, the method provided physiologically meaningful longitudinal strain curves with small dispersion among LV segments. On CRT patients, improved peak systolic longitudinal strain in the septum agreed with positive clinical response and reverse remodeling. Future work will include US-adapted similarity metrics and the extension of this registration framework to incorporate compounding strategies to address field of view issues in 3D-US sequences of heart failure patients with dilated LV.

Acknowledgments. This research has been partially funded by the Industrial and Technological Development Center (CDTI) under the CENIT-cvREMOD program and by the European Commission's project euHeart (FP7-ICT-224495). G. Piella and O. Camara were supported by the Ramón y Cajal Program from the Spanish Ministry of Science and Innovation. A. Doltra was supported by a Post-Residency Award from Fundació Clínic.

\section{References}

1. Grau, V., Becher, H., Noble, J.: Registration of multiview real-Time 3-D echocardiographic sequences. IEEE Trans. Med. Imag. 26(9), 1154-1165 (2007)

2. Elen, A., Choi, H., Loeckx, D., Gao, H., Claus, P., Suetens, P., Maes, F., D'hooge, J.: Three-dimensional cardiac strain estimation using spatio-temporal elastic registration of ultrasound images: a feasibility study. IEEE Trans. Med. Imag. 27(11), 1580-1591 (2008)

3. Kawagishi, T.: Speckle tracking for assessment of cardiac motion and dyssynchrony. Echocardiography 25(10), 1167-1171 (2008)

4. Ledesma-Carbayo, M., Kybic, J., Desco, M., Santos, A., Suhling, M., Hunziker, P., Unser, M.: Spatio-temporal nonrigid registration for ultrasound cardiac motion estimation. IEEE Trans. Med. Imag. 24(9), 1113-1126 (2005)

5. Beg, M., Miller, M., Trouvé, A., Younes, L.: Computing Large Deformation Metric Mappings via Geodesic Flows of Diffeomorphisms. Int. J. Comput. Vision 61(2), 139-157 (2005)

6. Rueckert, D., Aljabar, P., Heckemann, R.A., Hajnal, J., Hammers, A.: Diffeomorphic Registration using B-Splines. In: Larsen, R., Nielsen, M., Sporring, J. (eds.) MICCAI 2006. LNCS, vol. 4191, pp. 702-709. Springer, Heidelberg (2006)

7. Khan, A., Beg, M.: Representation of time-varying shapes in the large deformation diffeomorphic framework. In: ISBI, pp. 1521-1524. IEEE, Los Alamitos (2008)

8. Durrleman, S., Pennec, X., Trouvé, A., Gerig, G., Ayache, N.: Spatiotemporal atlas estimation for developmental delay detection in longitudinal datasets. In: Yang, G.Z., Hawkes, D., Rueckert, D., Noble, A., Taylor, C. (eds.) MICCAI 2009. LNCS, vol. 5761, pp. 297-304. Springer, Heidelberg (2009)

9. Edvardsen, T., Gerber, B.L., Garot, J., Bluemke, D.A., Lima, J.A., Smiseth, O.A.: Quantitative Assessment of Intrinsic Regional Myocardial Deformation by Doppler Strain Rate Echocardiography in Humans: Validation Against Three-Dimensional Tagged Magnetic Resonance Imaging. Circulation 106(1), 50-56 (2002) 